\title{
Calcul des seiches à partir du logiciel Refonde
}

\author{
Frédéric Pons ${ }^{1}$, Philippe Sergent ${ }^{2}$, Adrien Lambert ${ }^{3}$ \\ 1 Centre d'Etudes Techniques de l'Equipement \\ Département Risques Eau Construction / Service Hydraulique \\ Pôle d'Activité CS 7049913593 Aix-en-Provence cedex 3 \\ frederic.pons@developpement-durable.gouv.fr \\ 2 Centre d'Etudes Techniques Maritimes et Fluviales \\ 2 Bd Gambetta 60321 COMPIEGNE CEDEX \\ philippe.sergent@developpement-durable.gouv.fr \\ 3 Geoscience Montpellier/INPG HMG LEGI (Grenoble) \\ CC 60 Université Montpellier II 34095 MONTPELLIER CEDEX 5 \\ adrien.lambert@gm.univ-montp2.fr
}

\section{Résumé :}

Les phénomènes de seiches se manifestent de diverses manières dans les zones portuaires ou les baies. Un premier point sur les connaissances des gênes occasionnées par ces seiches a été fait dans le cadre d'une enquête lancée auprès des grands ports. Deux modules de calcul inclus dans le logiciel Refonde ont ensuite été utilisés sur deux sites dans le but de déterminer lequel de ces deux modules est le plus approprié pour la compréhension des seiches.

\begin{abstract}
:
Seiches appear with different forms and different consequences in French harbours and bays. An inquiry on national harbours has been done to know the effects and the problems linked to seiches. After, Refonde software was used on two sites to know the most interesting module for the understanding of the seiche consequence.
\end{abstract}

Mots-clés :

Seiches - Modélisation - Berkhoff

DOI: 10.5150/jngcgc.2008.074-P $\quad$ (disponible en ligne - http://www.paralia.fr - available online) 


\section{$1 \quad$ Introduction}

Les seiches sont provoquées par la "résonance» dans un bassin portuaire ou une baie d'une onde incidente de période allant de 20 secondes à plusieurs minutes. Cette période propre est principalement fonction des caractéristiques géométriques du domaine de résonance.

Afin de mieux comprendre le phénomène de seiche et de connaître ses effets sur les principaux ports français métropolitains, un questionnaire a été adressé à certains ports français. Les réponses liées à ce questionnaire et notre connaissance sur le sujet (disponibilité de données...) nous ont permis de retenir deux sites d'études, à savoir, le site de la baie d'Agay (LAMBERT, 2006) et le site du Port de Bayonne.

Afin d'étudier ces deux sites, les théories du logiciel Refonde, de son module d'agitation et de son module seiche, sont rappelées succinctement.

\section{$2 \quad$ Analyse d'une enquête sur les seiches en différents ports francais}

Nous avons eu un retour de 11 réponses sur 24 ports questionnés. Les questionnaires étaient basés sur trois points principaux :

- La connaissance locale des variations de niveau d'eau liées aux tsunamis.

- La connaissance locale des seiches.

- La disponibilité des données pour une analyse numérique des seiches.

Concernant la problématique des seiches, le mot « seiche » est connu à $90 \%$ de la communauté et seuls deux ports nous ont indiqué que des seiches apparaissaient dans leur infrastructure, à savoir le port de Cherbourg et le Port de Bayonne. Ce dernier semble être vraiment le plus impacté par le phénomène en France.

Le phénomène de seiche à Cherbourg ne semble pas gêner l'activité portuaire tandis qu'à Bayonne le phénomène est fort contraignant. L'amplitude est de l'ordre d'un mètre et les périodes sont mal connues ou en gamme de période très large d'après les réponses. Les conséquences à Bayonne sont des chocs entre bateaux, des ruptures d'amarres, la maintenance en alerte d'un remorqueur dédié à ce problème et la mise en place de matériel spécifique gênant l'activité portuaire à quai.

A travers nos diverses lectures ou discussions orales, il a été également recensé des problématiques de seiches sur les ports suivants :

- Sète avec des périodes autour de 15 min (GOMI, 1998).

- Port-Tudy (Groix) avec des amplitudes de l'ordre de 1 à 1,5 m, une période de l'ordre de 4,5 min. Les seiches se produisent en moyenne 70 jours par an et entraînent la fermeture du Port entre 4 et 5 jours par an (FLETOUR, 2004).

- Dieppe où l'amplitude de la seiche est de l'ordre de 1 à $1,5 \mathrm{~m}$. 
- Dunkerque où une rupture importante du talus du QPO avait été imputée à une seiche à la fin des années 1980 .

- Marseille (Golfe de Fos) où une seiche d'amplitude de $1 \mathrm{~m}$ peut apparaître en moyenne une fois tous les 5 ans.

Concernant la disponibilité des données, le port de Bayonne s'est montré fort intéressé par cette étude et nous a fourni l'ensemble des données en sa possession en indiquant par avance qu'il ne disposait pas de mesure des seiches fiables en amplitude et en période.

La baie d'Agay (LAMBERT, 2006) située sur le littoral Varois entre le Cap Dramont à l'Ouest et la pointe de la Baumette à l'Est, a aussi été choisie. Des mesures fines de l'ensemble des paramètres hydrodynamiques sur le site et des mesures d'oscillations de la surface de l'eau assimilables à des seiches sont disponibles.

Cette enquête et recensement n'ont pas pour objectif d'être exhaustifs pour l'heure sur les problématiques d'ondes de périodes comprises entre celles de la houle et celles de la marée dans les ports. De plus, tant que des dommages ou gênes ne sont pas occasionnés par les tsunamis ou les seiches, les autorités portuaires se préoccupent peu de ces phénomènes. Des mesures relatives à ces phénomènes commencent à être disponibles avec la mise en place d'enregistrement marin au pas de 10 minutes dans le cadre du réseau RONIM (SCHINDELE, 2008). Cependant, ces mesures n'ont pas encore un pas d'enregistrement suffisant pour capter ces ondes en amplitude et en période.

\section{Rappel théorique des seiches et de l'application sur Refonde}

Les ondes de seiches font partie de la famille des ondes de gravité. En pratique, ces ondes longues sont très peu cambrées et ne déferlent pas. Elles sont modifiées par réfraction et diffraction d'autant plus que leur période est grande. Elles sont aussi réfléchies avec très peu de dissipation et ce d'autant moins que leur période est grande.

\subsection{Module Agitation}

Le logiciel Refonde qui résout l'équation de Berkhoff par éléments finis est utilisé. Nous avons mis en œuvre une étude de l'agitation avec toute une gamme de paramètres différents d'ondes, à savoir, cote moyenne du plan d'eau, hauteur de l'onde incidente, période de l'onde et direction sur le bord ouvert du domaine. Les frontières terrestres sont toujours considérées totalement réfléchissantes. Ce type de calcul par une méthode de balayage en fréquence ou en période est utilisé assez couramment (GOMI, 1998). L'objectif est d'ensuite relever la hauteur d'onde maximale atteinte sur le domaine (en vérifiant qu'elle ne constitue pas un 
artefact numérique) et de la comparer avec la hauteur de l'onde incidente injectée. Pour effectuer cette comparaison une amplification est définie comme étant le rapport de la hauteur d'onde maximale dans le domaine sur la hauteur d'onde incidente.

\subsection{Module Seiche}

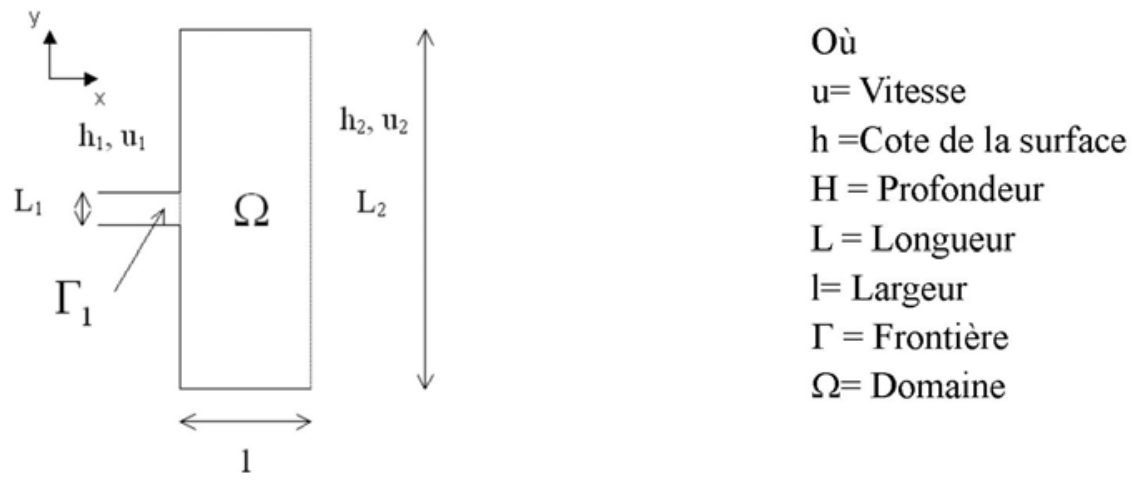

Figure 1 : Schéma d'expansion infinie en mer ouverte

Le module Seiche considère certaines extrémités du domaine comme une expansion infinie en mer ouverte. Sur cette expansion infinie, l'équation des ondes sur fond plat peut s'écrire :

$$
\frac{\partial h}{\partial t}+H \nabla \vec{u}=0 \quad \text { et } \quad \frac{\partial \vec{u}}{\partial t}+g \vec{\nabla} h=0
$$

Intégrées sur le domaine $\Omega$, ces deux équations donnent :

$$
\int_{\Omega} \frac{\partial h}{\partial t}=-H \int_{\Gamma} \vec{u} \cdot \vec{n}=-H\left(u_{2} L_{2}-u_{1} L_{1}\right) \quad \int_{\Omega} \frac{\partial \vec{u}}{\partial t} \cdot \vec{e}_{x}=-g \int_{\Gamma} h \cdot \vec{n} \cdot \vec{e}_{x}=-g L_{2}\left(h_{2}-h_{1}\right)
$$

Sous l'hypothèse des ondes longues et d'une expansion brusque $\frac{l}{\lambda} \approx 0$, on en déduit les relations suivantes avec $\mathrm{h}_{0}$ et $\overrightarrow{\mathrm{u}}_{0}$ les amplitudes moyennes de la hauteur de houle et de la vitesse sur le domaine $\Omega, l$ la largeur du domaine $\Omega$ et $\lambda$ la longueur d'onde.

$$
\int_{\Omega} \frac{\partial h}{\partial t} \propto \mathrm{h}_{0} c L_{2} \frac{l}{\lambda} \approx 0 \quad \int_{\Omega} \frac{\partial \vec{u}}{\partial t} \propto \overrightarrow{\mathrm{u}}_{0} c L_{2} \frac{l}{\lambda} \approx 0
$$

A partir des équations (2) et (3), on obtient les relations suivantes :

$$
u_{1}=u_{2} \frac{L_{2}}{L_{1}} \text { et } h_{1}=h_{2}
$$

Comme la vitesse et la hauteur de houle sont nulles côté mer, on en déduit que la hauteur de houle est aussi nulle à la frontière $\Gamma_{1}$ par continuité. La vitesse en revanche est discontinue et non nulle à la frontière $\Gamma_{1}$ car le rapport $L_{2} / L_{1}$ est 
infini.

Les conditions aux limites appliquées au potentiel de vitesse $\psi$ sont donc les suivantes :

- $\quad$ Expansion infinie en mer ouverte : $\psi=0$

- Frontière réfléchissante : $\quad \frac{\partial \psi}{\partial n}=0$

Le calcul des seiches consiste alors à déterminer la suite infinie de valeurs propres $k_{n}$ et de vecteurs propres $\psi_{n}$ satisfaisant les conditions aux limites (5) et (6) et solutions de l'équation de Berkhoff :

$$
\nabla\left(c c_{g} \nabla \psi_{n}\right)+c c_{g} k_{n}^{2} \psi_{n}=0
$$

\section{$4 \quad$ Applications}

Les deux sites d'études, à savoir, le site de la baie d'Agay (LAMBERT, 2006) et le site du Port de Bayonne présenté ci-dessous ont chacun un maillage par longueur d'onde (10 nœuds pour une période de 100 secondes).

\subsection{Baie d'Agay}

La baie d'Agay se situe dans le Var. Elle a fait l'objet d'un suivi de paramètres d'agitation au cours d'une thèse (LAMBERT, 2006). Une expérimentation a utilisé des capteurs de pression dans la plage et ces capteurs ont mesuré des oscillations peu amorties d'une onde de période de 577 secondes.

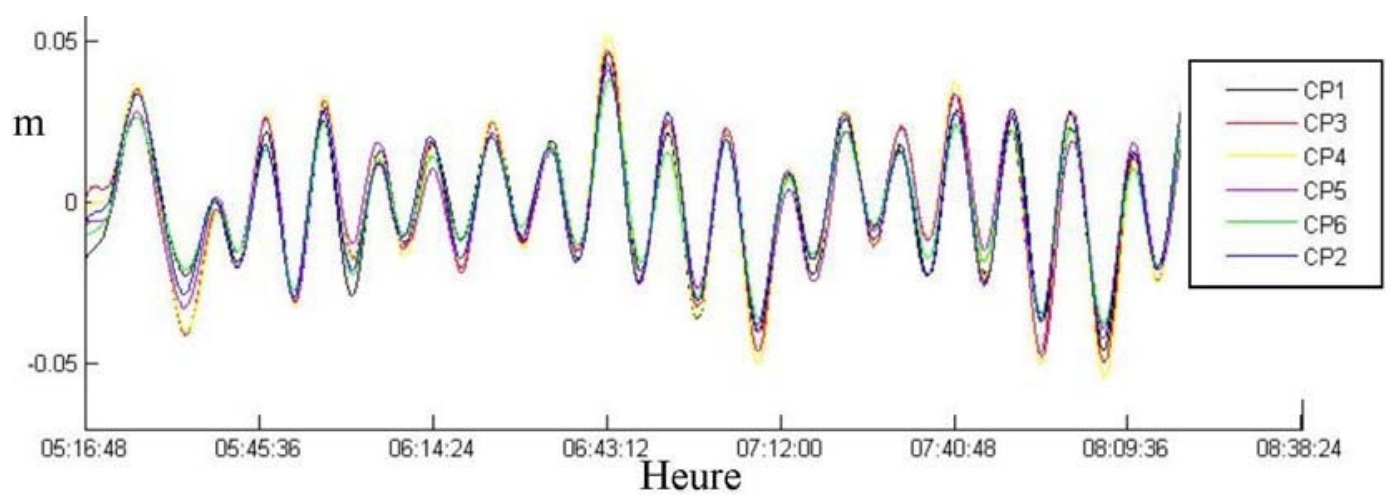

Figure 2 : Amplitude des signaux filtrés sur 6 piézomètres positionnés sur la plage émergée de la baie d'Agay le 20/01/2005 matin

\subsubsection{Module Seiche}

Le module seiche nécessite la délimitation plus ou moins arbitraire du domaine semi isolé par une frontière ouverte. Nous avons choisi dans le cas de la baie d'Agay la largeur la plus étroite pour situer cette frontière (comparaison de la figure 3 et de la figure 5). 


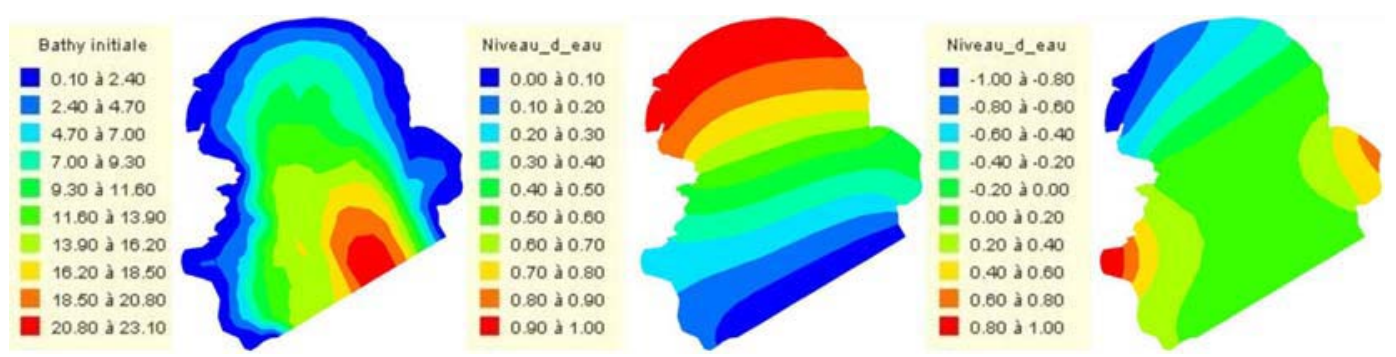

Figure 3 : Bathymétrie en $m$ (gauche) et forme des seiches pour une cote d'eau de $0.01 \mathrm{~m}$ NGF et des périodes de $435 \mathrm{~s}$ (milieu) et $218 \mathrm{~s}$ (droite) sur la baie d'Agay

Le module seiche fournit comme résultats la valeur de $435 \mathrm{~s}$ pour la seiche la plus longue à rapprocher de la mesure à $577 \mathrm{~s}$, et de $218 \mathrm{~s}$ pour la seconde.

\subsubsection{Module agitation par balayage}

Le balayage en période sur la baie d'Agay donne des résultats d'amplification très proches quelle que soit la hauteur de l'onde incidente testée de 0,01 à $0,6 \mathrm{~m}$. Le résultat de la période de la seiche principale (figure 4) est de l'ordre de $560 \mathrm{~s}$, valeur totalement comparable avec les 577 s mesurés pendant les expériences sur la plage émergée.

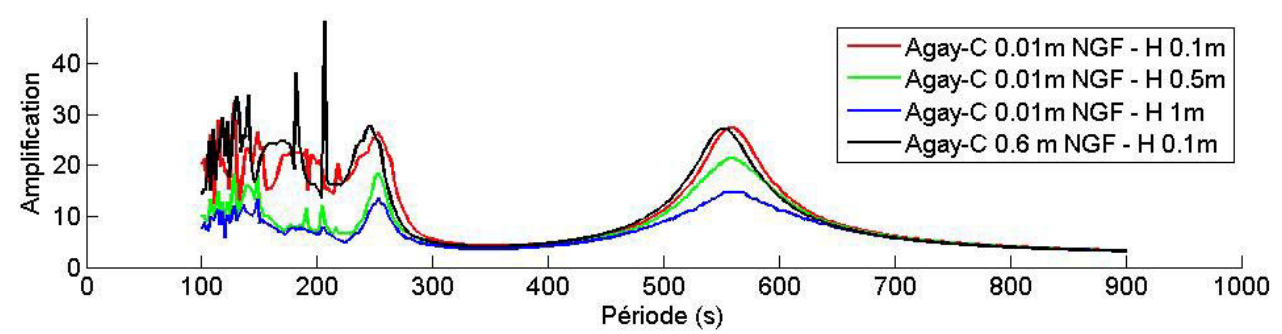

Figure 4 : Amplification en fonction de la période pour la baie d'Agay, pour quatre conditions simulées

La seiche secondaire se trouve vers $250 \mathrm{~s}$. Les autres oscillations entre 100 et $200 \mathrm{~s}$ sont des oscillations très localisées de certaines parties du plan d'eau.


Figure 5 : Bathymétrie en $m$ (gauche) et agitation pour une cote d'eau de $0.01 \mathrm{~m}$ $N G F$, une hauteur de houle de $0.1 \mathrm{~m}$, et des périodes de $560 \mathrm{~s}$ (milieu) et $250 \mathrm{~s}$ (droite) sur la baie d'Agay

Le choix du critère de déferlement a peu d'incidence sur les résultats (non 
présenté dans le texte) et l'écrêtage est limité aux faibles profondeurs. Ceci est visible par comparaison des figures 3 et 5 en bordure des frontières terrestres.

\subsection{Port de Bayonne}

Le port de Bayonne se situe dans l'estuaire de l'Adour, à cheval sur les deux départements des Landes et des Pyrénées Atlantiques. Les bathymétries du port de Bayonne sont issues de levés du port avec un point tous les mètres, du contour officiel du port et d'autres levés disponibles. Ces levés en zéro hydrographique ont été simplifiés avec 1 point tous les $25 \mathrm{~m}$ dans l'estuaire et tous les $100 \mathrm{~m}$ sur l'océan.

La représentation bathymétrique (figure 6) du modèle du port de Bayonne est montrée avec une plage de valeur de -1 à 14 mètres et un zoom vers la zone d'intérêt du port de plaisance et de la partie aval touchées par les seiches.

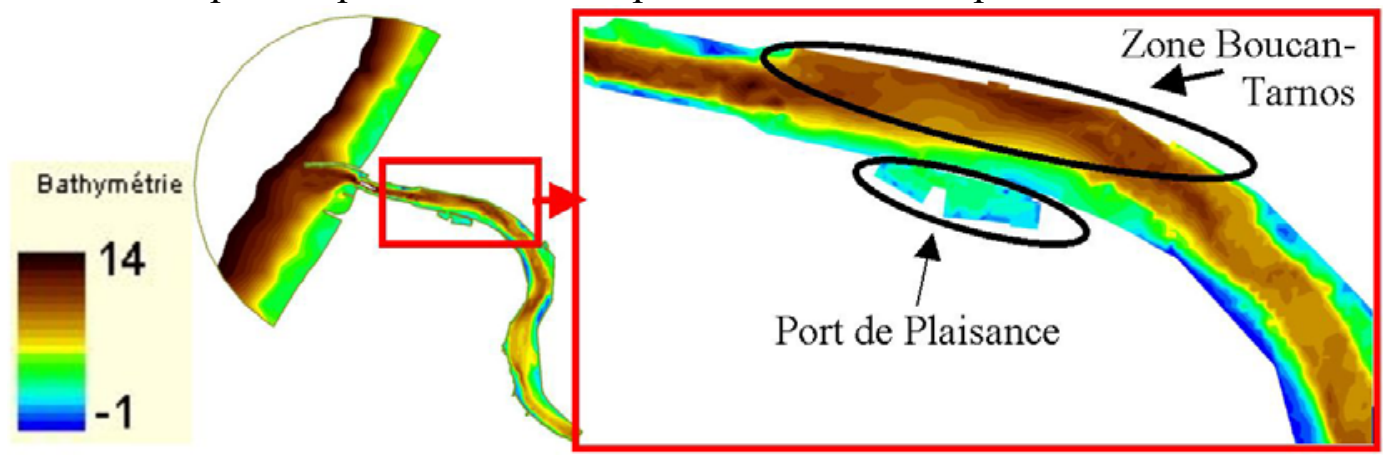

Figure 6 : Bathymétrie en $m$ du modèle de l'Adour

Les conséquences des seiches sont bien connues sur ce site depuis les années 1960. Les témoignages (notes et contacts oraux avec le service études et travaux maritimes et la capitainerie) font état de plusieurs fréquences de seiches :

- Une seiche agitant la partie aval de la rivière avec une période de $2 \mathrm{~min}$.

- Une seiche agitant un quai particulier avec une période de $1 \mathrm{~min}$.

- Une seiche agitant principalement le petit port de plaisance avec une amplitude de 1 à $2 \mathrm{~m}$ et une période de $2 \mathrm{~min}$ (ordre de grandeur) ou de 3 min avec une amplitude de l'ordre du mètre.

- Une autre seiche qui agite tout le port de 20 à $40 \mathrm{~min}$ et surtout la zone de Boucan-Tarnos.

Dans le jargon local, ces phénomènes portent le nom de « ressac ». Malgré des enregistrements qui montrent des seiches plus importantes à la pleine mer qu'à la basse-mer (liés probablement aux positions des marégraphes), les effets du « ressac » (gênes, dégâts) sont bien plus importants à basse mer qu'à pleine mer d'après l'ensemble des témoignages. Le filtrage de la marée sur les données du marégraphe au pas de temps de 10 minutes en 2007 montre une oscillation 
présente à la fois en basse mer et pleine mer mais ne permet pas de conclure.

\subsubsection{Module Seiche}

L'utilisation du module seiche nous fournit différentes périodes. La figure 7 montre les formes des seiches pour une cote de $0.75 \mathrm{~m} \mathrm{ZH}$. Ces formes sont identiques pour une cote d'eau de $4.75 \mathrm{~m} \mathrm{ZH}$ mais leur période est modifiée (tableau 1). La condition amont est une hauteur de houle nulle qui ne semble pas correcte compte tenu des résultats obtenus par la méthode d'agitation par balayage et présentés plus bas.

Tableau 1 : Période des seiches avec le module seiche sur l'estuaire de l'Adour

\begin{tabular}{|c|c|c|c|c|c|}
\hline Cote Eau & Mode 1 & Mode 2 & Mode 3 & Mode 4 & Mode 5 \\
\hline $0.75 \mathrm{~m} \mathrm{NGF}$ & $1541 \mathrm{~s}$ & $740 \mathrm{~s}$ & $474 \mathrm{~s}$ & $333 \mathrm{~s}$ & $298 \mathrm{~s}$ \\
\hline $4.75 \mathrm{~m} \mathrm{NGF}$ & $1080 \mathrm{~s}$ & $517 \mathrm{~s}$ & $333 \mathrm{~s}$ & $230 \mathrm{~s}$ & $195 \mathrm{~s}$ \\
\hline 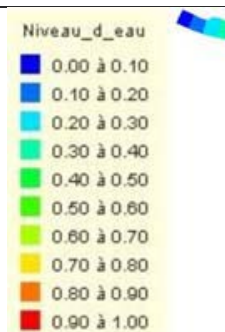 & &  & & 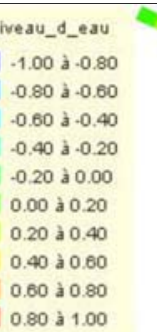 & \\
\hline
\end{tabular}

Figure 7 : Forme des seiches pour une cote d'eau de $0.75 \mathrm{~m}$ ZH et des périodes de $1538 \mathrm{~s}$ (à gauche), $739 \mathrm{~s}$ (milieu), $474 \mathrm{~s}$ (à droite) sur l'estuaire de l'Adour

\subsubsection{Module Agitation par balayage}

La méthode par balayage testée avec le module agitation (figure 8 en prenant une condition sur la frontière ouverte absorbante montre quatre grands groupes.
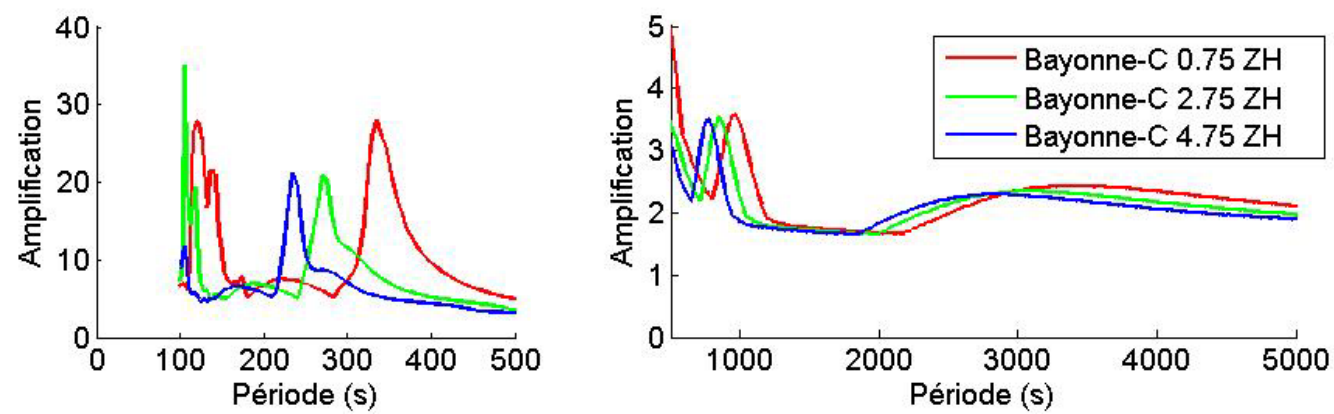

Figure 8 : Amplification en fonction de la période sur l'estuaire de l'Adour

Le premier, autour de 100 à $150 \mathrm{~s}$ correspond à des artefacts numériques dans certains coins du port très localisés. Le deuxième groupe entre 235 et $335 \mathrm{~s}$ correspond à l'agitation dans le port de plaisance. L'amplification et la période de 
cette seiche est d'autant plus importante que le niveau d'eau diminue. Le troisième groupe entre 770 et $960 \mathrm{~s}$ correspond à une agitation du port de plaisance et de la zone de Boucan-Tarnos. L'amplification n'est pas modifiée par le niveau d'eau, par contre, la zone impactée est plus importante à basse mer. Le quatrième groupe correspond à des périodes entre 2600 et 3500 s. L'amplification liée à ces ondes est bien plus faible.

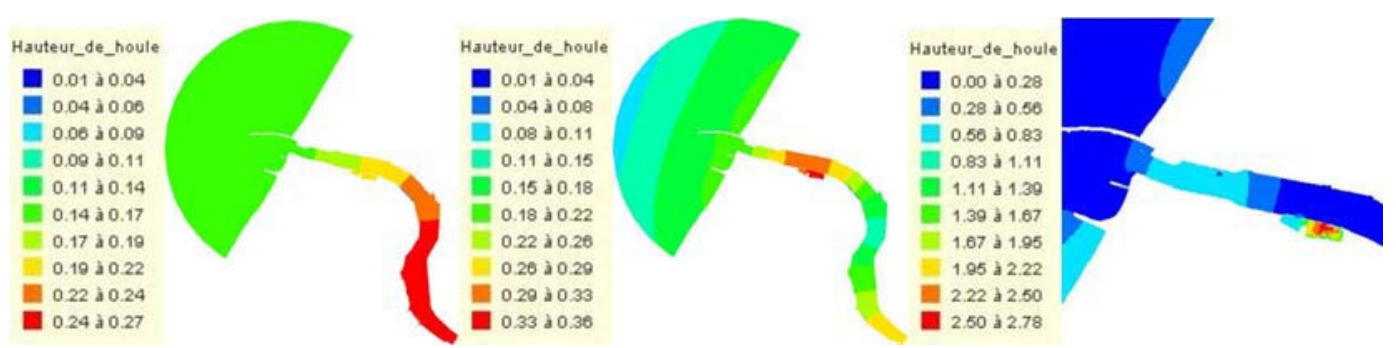

Figure 9: Agitation pour une cote d'eau de $0.75 \mathrm{~m}$ ZH et des périodes de $3400 \mathrm{~s}$ (à gauche), $960 \mathrm{~s}$ (milieu) et $335 \mathrm{~s}$ (à droite) sur l'estuaire de l'Adour

Trois types de seiches pour une cote d'eau de $0.75 \mathrm{~m} \mathrm{ZH}$ sont visualisables sur la figure 9 :

- Une seiche généralisée sur l'ensemble de l'estuaire de l'Adour dont le calcul est peut-être influencé par la position de la condition aux limites amont de l'estuaire où une frontière ouverte est imposée. Les principales limites du calcul sont d'une part la prise en compte d'un plan d'eau horizontal et d'autre part le fait de négliger les courants de marée et de l'Adour.

- Une seiche de période $960 \mathrm{~s}$ (16 min) avec un maximum de hauteur observé au niveau de la zone aval de l'estuaire comprenant le port de plaisance et la zone de Boucan-Tarnos.

- Une seiche de période de $335 \mathrm{~s}$ générée uniquement à l'intérieur du port de plaisance.

Ces trois seiches se retrouvent avec les mêmes formes pour des cotes d'eau plus élevées. Les périodes des seiches sont diminuées avec une augmentation $d u$ niveau d'eau. L'amplification est la même sauf pour celle touchant le port de plaisance où plus le niveau baisse, plus l'amplification augmente.

\section{$5 \quad$ Synthèse et Perspectives}

Les gênes occasionnées par les seiches dans les infrastructures portuaires ne sont pas exceptionnelles mais apparaissent de manière récurrente sur l'ensemble des façades métropolitaines. Une enquête lancée en 2007 va être poursuivie afin de compiler plus d'information sur ce sujet.

Le logiciel Refonde permet d'étudier les périodes de seiches dans les ports avec 
une méthode par balayage en période basée sur le module agitation ainsi qu'avec une méthode directe appelée seiche. La méthode seiche est facile et rapide à mettre en œuvre. Elle génère tous les modes en un seul calcul de manière systématique. Elle fournit la période et le mode propre mais ne donne pas le facteur d'amplification. Elle nécessite de plus le positionnement plus ou moins arbitraire d'une frontière où le potentiel est imposé à zéro et dont le choix influence fortement les résultats.

La méthode plus habituelle par balayage en période permet d'obtenir des résultats plus fiables avec un facteur d'amplification pouvant être évalué. Elle nécessite cependant un temps de calcul plus important. Cette méthode peut aussi parfois ne pas relever certains modes lorsque le pic est étroit, le pas en période de balayage trop grossier ou l'amplification de ce mode peu importante.

Les deux méthodes sont donc complémentaires : la méthode seiche permettant de « dégrossir » rapidement le problème et la méthode par balayage " d'affiner » les calculs.

Ce travail a permis de mieux appréhender les conséquences et le calcul des périodes de seiches mais nécessite un approfondissement à l'avenir des effets générateur de ces ondes.

Il est également envisagé d'utiliser sur le cas du port de Bayonne un modèle de Saint-Venant pour pouvoir mieux intégrer l'effet des courants et de plan d'eau non constant.

\section{$6 \quad$ Références bibliographiques}

${ }^{1}$ FLETOUR J-B. (2004). Étude relative aux phénomènes de seiches à Port-Tudy (Groix). Mémoire de stage ENSIETA, Conseil Général du Morbihan, 98 p.

${ }^{2}$ GOMI P. (1998). Les seiches. Mémoire de Fin d'Etudes au Service Technique Central des Ports Maritimes et des Voies Navigables, Ecole Nationale des travaux Publics de l'Etat, 84 p plus annexes.

${ }^{3}$ LAMBERT A. (2006). Protection durable du littoral varois : fonctionnement hydrosédimentaire de plages microtidales équipées de systemes de drainage. Thèse, Université Aix-Marseille I - Université de Provence, U.F.R des Sciences Géographiques et de l'Aménagement, $379 \mathrm{p}$.

${ }^{4}$ SCHINDELE F., HEBERT H. (2008). La participation de la France dans la mise en place du réseau de surveillance du niveau de la mer en Méditerranée Occidentale et dans l'Atlantique Nord-Est. Lettre du Réseau RONIM, UMR CNRS 6250 LIENSS, Université de La Rochelle, Mars 2008 pp 4-5. 